\title{
Analysis of Technology Spillover between Vertical Industry--From Chinese Manufacturing Evidence
}

\author{
Mengdan Shi Yaping Fu
}

School of Finance, Guizhou University of Finance and Economics, Guiyang Guizhou 550025, China

\begin{abstract}
According to Mario Forni \& Sergio Paba (2002) method, using the input-output table of the relationship between the upstream and downstream of 35 industry recognition, using 2005-2007 test the empirical data of industrial enterprises upstream $\mathrm{R} \& \mathrm{D}$ investment impact on the downstream industry productivity, there are significant technology spillover effect of the downstream industry, further inspection found by not only bring technology spillover, downstream industry productivity, but also increase the downstream industry labor wages, namely spillover benefits for the enterprise and employees to share.
\end{abstract}

\section{Keywords}

Technology spillover; Total factor productivity; Vertical industry

\section{垂直产业之间的技术溢出分析 \\ 一来自中国制造业的证据}

\author{
石孟丹 傅亚平 \\ 贵州财经大学金融学院, 贵阳 550025, 中国
}

摘要：本文借鉴 Mario Forni \& Sergio Paba（2002）的方法，利用投入产出表对 35 个产业的 上下游关系进行识别, 运用 2005-2007 年工业企业数据实证检验上游研发投入对下游产业生 产率的影响, 研究发现上下游产业间存在显著的技术溢出效应, 进一步的检验发现, 由技术 溢出不仅带来的下游产业生产率的提升, 还会增加下游产业劳动工资, 即溢出效应的收益为 企业与员工分享。

关键词: 技术溢出; 全要素生产率; 垂直产业 


\section{1. 引言}

在企业生产中，技术几乎与资本、 劳动具有同等重要的地位, 成为生产 过程中不可或缺的要素。然而, 与其 他生产要素不同, 技术是知识的某种 载体, 由于知识的非竞争与非排他性 而产生了溢出效应。自 Romer（1986） 将技术作为生产函数的独立生产要素 纳入内生经济增长模型以来, 关于研 发投入与知识、技术溢出等话题的研 究就逐渐成为学术界关注的焦点之一。

\section{2. 文献综述}

国内外关于技术溢出的研究文献 大致可以划分为两个层面:一是在宏观 经济研究的框架下, 研究技术溢出在 经济增长过程中的贡献。如 Romer

( 1990 ) , Grossman \& Helpman (1991a) , Barro \& Salai Martin （1997）在内生经济增长框架下, 探 讨国际间技术扩散对经济增长的重要 作用。Coe\&Helpman（1995）基于 22 个发达国家与 77 个发展中国家数据, 发现了发达国家的 R\&D 的投入对其贸 易伙伴国的发展中国家 TFP 具有显著 正向贡献的证据。二是基于企业生产 函数和企业微观数据研究技术在行业 内部以及行业之间的溢出效应。较早 地关注某个行业的研发投入可以对其 他行业生产率产生影响的是 Griliches (1979)。Los（1997）认为 知识溢出分为两种类型：一是行业内 部企业之间通过模仿使得整个行业技 术水平得到提升, 二是认知创新的溢 出过程（idea-creating）, 比如自然 学科公开发表的论文会对企业研发过 程提供指引。Los（2000），潘文卿 （2012）分别利用美、中两国的投入 产出表的直接消耗系数构造角余弦的 方法表征不同产业间的技术距离，从
而把工业分为 4 个门类测度技术溢出 效应, 相比较 4 个工业部类之间, 相 似度更高的工业部门内部的技术溢出 效应更强。Mario Forni \& Sergio Paba（2002）通过投入产出表识别制 造业之间的上下游垂直关系, 运用意 大利工业企业的数据, 考察研发投入 对其他行业生产率的影响, 发现了产 业间的投入产出关系在技术传导中的 关键作用。国内关于技术溢出的研究 文献大多是关于该领域的, 陈涛涛 (2006)，沈坤荣 (2009) 重点研究了 外资对内资同行业的水平溢出, 陈羽 (2006), 姜瑾 (2007)，许连和 (2007) 关注 FDI 对内资产业间的垂直溢出效 应, 并发现垂直溢出效应高于水平溢 出效应。综合前面对文献的梳理, 可 以发现基于企业微观层面数据, 从整 个工业部门角度研究行业间技术溢出 的文献较少。本文拟根据 2005-2007 年 35 个部门的规模以上工业企业数据, 分析、检验上游研发投入对下游行业 生产率的影响。

\section{3. 垂直产业间技术溢出理论及垂直产 业结构识别}

\section{1. 垂直产业间技术溢出理论}

知识的溢出过程是不同主体之间 通过直接或间接的方式进行交流中， 所发生的无意识的传播过程, 这一传 播过程受空间限制较低。技术作为特 殊的知识形态，溢出过程有其不同之 处，上下游行业交易的商品是产业间 技术传导的重要载体，有关垂直产业 间技术传导的重要理论总结如下:

Griliches (1979) 较早对技术扩散 进行分类与辨析, 按照技术溢出受益 者获得渠道, 他认为技术的溢出效应 分为知识溢出 ( knowledge spillovers) 和租金溢出效应 (rent spillovers）。知识的溢出效应一般 
源于公开的知识进步, 与知识溢出效 应不同, Griliches 所定义的租金溢出 效应，仅仅是源自于上下游企业所交 易的产品创新产生的，典型的例子是 计算机，若新款的计算机的计算速度 提升两倍, 新款计算机的价格会上 涨, 如此, 用户为单位计算效率的所 付出的成本就会下降。还有一种上下 游产业间技术溢出效应的可能渠道 是, 下游企业为了可以自由地运用他 们可获得的知识, 可能会追加研发投 入。从上述技术传导途径的归纳可以 发现, 上下游产业间的产品交易可能 不仅是租金溢出效应的重要载体, 还 可能是下游产业技术进步的催化剂。

\section{2. 垂直产业结构的识别}

本文运用 Mario Forni \& Sergio Paba（2002）的方法通过对 1997 年、 2002 年、 2007 年投入产出表界定产业 部门中上下游关联。首先, 按照工业 企业数据库 2 位代码将 38 个部门, 然 后，将工艺及其他制造业、废弃资源 及废旧材料回收加工也、水的生产和 供应业 3 个部门去掉, 因为这 3 个部 门数据缺失过多, 对后续计量计算带 来困难。第二, 计算每个部门中间投 入占其总中间投入的比例 $\lambda$, 如果 $i$ 部 门的中间投入 $j$ 所对应的比值 $\lambda_{j}$ 在 3 年的投入产出表中均超过 $5 \%$, 则界定 部门 $j$ 为 $i$ 部门的上游产业。

从计算结果看, 一般产业部门所 消耗的上游部门的比例变化不大, 上 游产业部门在投入产出表上基本集中 在对角线附近（见附表 1)，除了化学 原料及化学制品制造业、黑色金属冶 炼及延压工业、通用设备制造业 3 个 产业部门。由此, 可以发现这 3 个部 门对其他产业部门的关联度更高。

\section{4. 模型的设定与指标构造}

一般关于技术溢出的研究, 都是 将 R\&D 投入作为一个生产要素放入生 产函数中，通过估计 R\&D 与产出之间 的关系来测度 R\&D 的贡献, 如式 (1 所 示) :

$$
Y_{i j t}=f\left(K_{i j t}, L_{i j t}, R D_{i j}, I R D_{j}\right)
$$

其中, $Y_{i t}$ 表示行业 $j$ 中某企业 $i$ 在 $t$ 期的产出, $K_{i j t}$ 与 $L_{i j t}$ 分别表示企 业生产过程中资本与劳动投入, $R D_{i j}$ 表示生产过程中企业的研发投入, $I R D_{j}$ 表示行业的间接研发投入，此处 表现为上游行业的总研发投入。

然而, 全要素生产率 ( TFP ) 是 表征生产率高低的直接指标变量, 故 而, 本文采用行业 $T F P_{i j}$ 代替行业产出 $Y_{j}$ 测度上游行业研发投入 $I R D_{j}$ 对本 产业部门生产率的贡献。文章采用对 行业内企业 $T F P_{i j}$ 加权的方法计算:

$$
\ln T F P_{j}=\sum_{i} \omega_{i} \ln T F P_{i j}
$$

其中 ${ }^{\omega_{j}}$, 权重 ${ }^{t}$ 取企业工业增加 值占本行业工业增加值总额的比例, TFP ${ }_{i j}$ 通过 OP 方法对下式的回归估计 计算:

$$
\ln T F P_{i j t}=\ln Y_{i j t}-\ln K_{i j t}-\ln L_{i j t}
$$

其中, $Y_{i j t}$ 表示企业在期的用工业出 厂价格指数折算后的工业增加值, $k_{i j t}$ 表示 ${ }^{t}$ 期用固定资产折算价格指数折算 后的固定资产， $L_{i j t}$ 用企业全年平均从 业人数计算。在使用 OP 计算过程中, 由于缺少企业每年投资的数据, 本文 借鉴宏观领域计算投资的方法, 公式 计算:

$$
I_{i j t}=K_{i j t}-K_{i j t-1}+\sigma_{i j t}
$$


其中, $\sigma_{i j t}$ 表示 ${ }^{t}$ 期经过固定资产 折算价格指数校正过的企业折旧。由 生产函数发现, 行业 ${ }^{T F P}{ }_{j}$ 是关于本行 业研发 $R D_{j}$ 与上游产业研发的函数:

$$
\ln T F P_{j t}=h\left(R D_{j t}, I R D_{j t}\right)
$$

其中,

$$
R D_{j t}=\sum_{i} \omega_{i t} R D_{i j t}
$$

$$
I R D_{j t}=\sum_{k \neq j} \mu_{k t} R D_{k t}, \mu_{k} \text { 为产业的各上 }
$$
游产业部门中间投入品消耗比值计算得

$$
\mu_{k t}=\frac{\lambda_{k t}}{\sum_{l} \lambda_{l t}}
$$

到的权重, 即

。对于中间投

入品消耗比值 $\lambda_{k t}$ 除了 1997 年、 2002 年、 2007 年的数据可以直接从投入产出表直 接计算得到外，其他各年的数值借鉴潘 文卿（2012）运用投入产出表界定产业 相似度的方法。中间投入品消耗比值公 式如下:

$$
\begin{aligned}
& \lambda_{j i t}=\left(1-\frac{t-1997}{5}\right) \lambda_{j 1997}+\left(\frac{t-1997}{5}\right) \lambda_{i j 2002}, t=1998,1999,2000,2001 \\
& \lambda_{j i t}=\left(1-\frac{t-2002}{5}\right) \lambda_{i j 202}+\left(\frac{t-2002}{5}\right) \lambda_{i j 2007}, t=2003,2004,2005,2006
\end{aligned}
$$

对（5）式取线性估计形式:

$\ln T F P_{j t}=\alpha_{0}+\alpha_{1} I R D_{j t}+R D_{j t}+k_{j t}+e d u_{j t}+a_{j}+\varepsilon_{j t}$

其中, $k_{j t}$ 与 $e d u_{j t}$ 为控制变量。 $k_{j t}=\sum_{i} \omega_{i} \frac{K_{i j t}}{L_{i j t}}$

表示行业中人均资本存

$$
e d u_{j t}=\sum_{i} \omega_{i} \frac{e d u_{i j t}}{L_{i j t}}
$$

量,

表示用居民 消费价格指数折算过的行业平均的职工 教育投入。

本文的模型延续多数实证研究文献 的做法, 仅关注横截面固定效应。价格
折算指数均以 1990 年为基期, 数据来 源于国家统计局和 2005-2007 年工业企

\begin{tabular}{|c|c|c|}
\hline $\begin{array}{l}\text { VARIABLE } \\
\mathrm{S}\end{array}$ & $\begin{array}{l}(1) \\
\text { tfpss }\end{array}$ & $\begin{array}{l}\text { (2) } \\
\text { tfpss }\end{array}$ \\
\hline yjkffw & $\begin{array}{l}4.98 \mathrm{e}-07^{\star \star *} \\
{[1.07 \mathrm{e}-07]}\end{array}$ & \\
\hline L.yjkffw & & $\begin{array}{l}3.93 \mathrm{e}-07^{* *} \\
{[1.62 \mathrm{e}-07]}\end{array}$ \\
\hline yjkff_ns & $\begin{array}{l}-2.16 \mathrm{e}-08 \\
{[2.38 \mathrm{e}-08]}\end{array}$ & $\begin{array}{l}-9.58 \mathrm{e}-09 \\
{[5.75 \mathrm{e}-08]}\end{array}$ \\
\hline k_l & $\begin{array}{l}0.00430^{*} \\
{[0.00227]}\end{array}$ & $\begin{array}{l}0.00344 \\
{[0.00235]}\end{array}$ \\
\hline zgjyfns_I & $3.26 \mathrm{e}-07$ & $-2.76 e-06$ \\
\hline
\end{tabular}
业数据库。

\section{5. 模型估计与分析}

\section{1. 初步回归结果}

模型用固定效应（FE）进行回归， 采用稳健标准差校正过的估计结果如 下表 1 所示:

表 1

变量的符号预期基本相同, 第一 列是按照 (7) 式的估计结果, 可以看 出, 上游行业的研发投入在 $5 \%$ 水平上 对下游行业生产率产生显著的积极影 响, 上游研发投入每增加 1000 万元会 导致下游生产率提升约 5 个百分点, 其他可能影响下游生产率的控制变量 中 (除人均资本存量 ${ }^{k}$ 在 $10 \%$ 水平上显 著外) 并不显著。然而, 具有长期合 作关系的上下游企业，下游企业可能 会对双方交易的商品的某些瑕疵进行 抱怨, 并根据自己的使用需要提供某 些改进建议, 影响上游产业的研发投 入, 继而对于模型估计可能出现双向 联立内生性。基于综合考虑, 由于本 文采用的是年度数据, 采用滞后一期 的上游研发投入 ${ }^{L . I R D}$ 作为 $I R D$ 的替 
代变量带入模型重新估计。结果如表 （一）第 2 列所示，估计结果与之前 差别不大, 故第一列的估计结果由于 双向联立性产生的可能的内生性影响 并不大。

\section{2. 稳健性估计}

当整个市场达到均衡状态时, 劳动 的价格一一工资等于劳动的边际产出, 故而, 工资是企业生产效率高低的一个 反应。本文采用行业人均工资水平 wage 替代 $^{T F P}$ 重新进行估计, 结果 如表 2 前两列所示。

表 2

\begin{tabular}{|c|c|c|c|c|}
\hline $\begin{array}{l}\text { VARIABLE } \\
\mathrm{S}\end{array}$ & $\begin{array}{l}\text { (1) } \\
\text { Inwage_ I }\end{array}$ & $\begin{array}{l}\text { (2) } \\
\text { Inwage_ I }\end{array}$ & $\begin{array}{l}\text { (3) } \\
\text { Inwage_I }\end{array}$ & $\begin{array}{l}\text { (4) } \\
\text { Inwage_ I }\end{array}$ \\
\hline yjkffw & $\begin{array}{l}3.35 \mathrm{e}- \\
07^{* * *} \\
{[9.02 \mathrm{e}-08]}\end{array}$ & & $\begin{array}{l}1.42 \mathrm{e}-08 \\
{[9.96 \mathrm{e}-08]}\end{array}$ & \\
\hline L.yjkffw & & $\begin{array}{l}3.95 \mathrm{e}-07^{* * *} \\
{[9.91 \mathrm{e}-08]}\end{array}$ & & $\begin{array}{l}1.65 \mathrm{e}-07 \\
{[9.82 \mathrm{e}-} \\
08]\end{array}$ \\
\hline yjkff_ns & $\begin{array}{l}5.69 \mathrm{e}- \\
08^{* * *} \\
{[9.38 \mathrm{e}-09]}\end{array}$ & $\begin{array}{l}5.91 \mathrm{e}-08^{*} \\
{[3.11 \mathrm{e}-08]}\end{array}$ & $\begin{array}{l}7.08 \mathrm{e}- \\
08^{\star * *} \\
{[1.13 \mathrm{e}-08]}\end{array}$ & $\begin{array}{l}6.47 \mathrm{e}-08 \\
{[5.09 \mathrm{e}-} \\
08]\end{array}$ \\
\hline k_l & $0.00374^{\star}$ & $0.00232^{*}$ & 0.000970 & $\begin{array}{l}0.00030 \\
4\end{array}$ \\
\hline & [0.00194] & {$[0.00136]$} & {$[0.00120]$} & $\begin{array}{l}{[0.00077} \\
4]\end{array}$ \\
\hline zgjyfns_I & $\begin{array}{l}4.72 \mathrm{e}-06 \\
{[3.76 \mathrm{e}-06]}\end{array}$ & $\begin{array}{l}9.86 \mathrm{e}-07 \\
{[5.64 \mathrm{e}-06]}\end{array}$ & $\begin{array}{l}4.51 \mathrm{e}-06 \\
{[3.69 \mathrm{e}-06]}\end{array}$ & $\begin{array}{l}2.60 \mathrm{e}-06 \\
{[5.04 \mathrm{e}-} \\
06]\end{array}$ \\
\hline tfpss & & & $\begin{array}{l}0.645^{\star \star *} \\
{[0.104]}\end{array}$ & $\begin{array}{l}0.585^{\star \star \star} \\
{[0.0929]}\end{array}$ \\
\hline Constant & $\begin{array}{l}1.778^{\star * *} \\
{[0.147]}\end{array}$ & $\begin{array}{l}1.939^{\star * *} \\
{[0.117]}\end{array}$ & $\begin{array}{l}-1.159^{\star *} \\
{[0.448]}\end{array}$ & $\begin{array}{c}-0.806^{*} \\
{[0.453]}\end{array}$ \\
\hline $\begin{array}{l}\text { Observatio } \\
\text { ns }\end{array}$ & 105 & 70 & 105 & 70 \\
\hline R-squared & 0.383 & 0.309 & 0.739 & 0.660 \\
\hline $\begin{array}{l}\text { Number of } \\
\text { cyid }\end{array}$ & 35 & 35 & 35 & 35 \\
\hline
\end{tabular}

回归结果符号符合预期, 本文所 关注的核心变量 $I R D$ 是高度显著的, 表明上游行业每增加 1000 万元研发投 入, 下游行业员工薪水平均上涨约 3.35 个百分点。值得注意的是, 企业 对员工教育投入 $e d u$ 无论对工人工资 还是对要素生产率的影响都不显著, 表明我国企业的职工教育投入在提升 员工生产技能方面，只是具有良好的 愿望, 员工的生产能力可能更多依靠 在生产过程中的学习。此外, 结合表

（一）、（二）估计结果，人均资本 存量对生产率的影响在 $10 \%$ 水平上是显 著的, 人均资本存量增大单位劳动的 生产效率可能越高, 这验证了经济学 生产函数技术是凸性的特征。

本文第三、第四列检验下游行业劳 动从上游研发投入中获益的传导途径, 由回归结果可发现, 当在回归方程右边 加入要素生产率变量, 上游研发投入 $I R D$ 因素变得不显著，模型的拟合优 度大幅提升。说明生产效率确实是影响 工资水平的最重要的因素, 在均衡状态 下, 工资是要素生产率合适的替代变量。

\section{6. 基本结论}

垂直产业间的技术溢出是企业间 技术溢出效应的延伸，上下游产业间 发生联系的重要桥梁是上游产业提供 给下游产业生产过程中所需要的原料 或是生产设备, 交易的产品是技术溢 出的重要载体。本文利用投入产出表 对 35 个工业部门间的上下游关系进行 识别, 发现上游行业的研发投入对下 游产业的生产率具有显著的积极作用。 本文的研究同时具备一定的政策含义, 政府在向企业研发提供补贴或减税等 优惠措施时，上游行业的研发投入多 少可能政府重点关注的区域。 


\section{参考文献}

[1] 许和连、魏颖绮、赖明勇、王晨刚, “外商直接投资的后向链接溢出效 应研究”《管理世界》2007 第 4 期.

[2] 潘文卿、李子奈、刘强, “中国产 业间的技术溢出效应：基于 35 个 工业部门的经验研究” 《经济研究》 2011 第 7 期。陈涛涛、陈娇, “行业增长因素与我国 FDI 行业 内溢出效应” 《经济研究》2006 第 6 期.

[3] 沈坤荣、李剑，“企业间技术外溢 的测度” 《经济研究》2009 第 4 期.

[4] Romer, P. , Endogenous Technology Change, Journal of Political Economy , 1990, 98( 5), 71 )102.

[5] Coe, D. and H elpman, E. / Internationa1 R\&D $\mathrm{Sp}$ illovers. 0European E- conom ic Review, 1995, Vo.1 39, pp. 859- 887.

[6] Griliches, Zvi, 1979, "Issues in Assessing the Contribution of Research and Development to Productivity Growth" , The Bell Journal of Economics , 10, 92116.

[7] Grossman G.M, Helpman E (1991a) Innovation and growth in the global economy. MIT Press, Cambridge MA

[8] Jeffrey I. Bernstern 1989 , " Research and Development and Intraindustry pillovers: An Empirical Application of Dynamic Duality" The Review of Econo- mic Studies, Vol. 56, No. 2 (Apr., 1989), pp. 249267.

[9] Los, B. (1997), "A Review of Interindustry Technology Spillover Measu-rement Methods in Productivity Studies", mimeo.

[10] Barro, R. , and X. Sala--i Mart in, / T ech nological Dif fusion, onvergence, and Growth0, J ournal of Economi c Gr ow th , 1997, 2( 1), 1 ) 26.

[11]Los B. , 2000 , "The Empirical Performance of a New Inter-industry Technology Spillover Measure", in Saviotti, P.P. and Nooteboom, B. ( Eds) Techno- logy and Knowledge, 118-151 , Edward Elgar , Cheltenham .

[12] Mario Forni and Sergio Paba (2002) "Spillovers and the Growth of Local Industries" The Journal of Industrial Economics, Vol. 50, No. 2 (Jun., 2002), pp. 151-171.

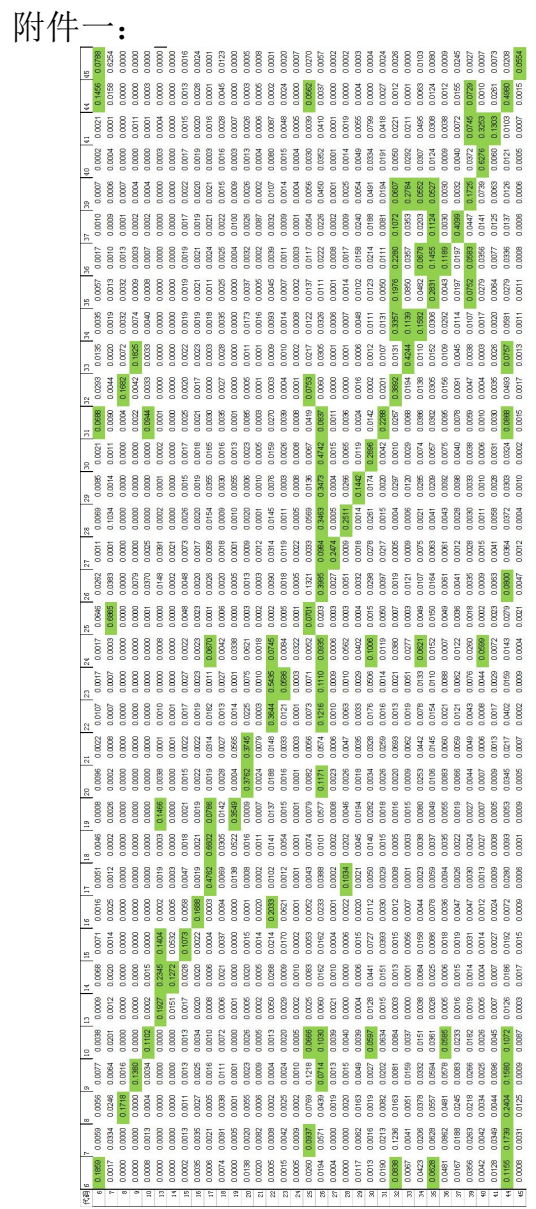

